\title{
LES RÉSEAUX SOCIAUX NUMÉRIQUES OFFRENT-ILS UNE ALTERNATIVE 2.0 AUX PRATIQUES TRADITIONNELLES DE MOBILISATION COLLECTIVE DANS LE MONDE DU TRAVAIL?
}

\author{
Caroline Sauvajol-Rialland $^{1}$
}

En France en 2011, trois entreprises du secteur de la grande distribution et du secteur du prêt-à-porter (Monoprix, Cora et Kookaii) ont fait l'objet de mobilisations « éclair » menées sur les réseaux sociaux à la suite desquelles deux d'entre elles ont renoncé aux décisions managériales qu'elles avaient prises (licenciements). Au-delà des différences de formes (déjà étudiées et liées aux technologies utilisées), existe-t-il une véritable différence de nature entre les formes de mobilisation sur les réseaux sociaux numériques et les formes traditionnelles de mobilisation collective dans le monde du travail ? L'objectif est de montrer l'apparition d'une nouvelle forme de mobilisation collective dans le monde du travail, spécifique dans sa nature et dans ses modalités. Compte tenu du caractère extrêmement récent des événements décrits, nous avons choisi une démarche descriptive et exploratoire, ainsi qu'une analyse de presse et une revue de la littérature existante. Plusieurs spécificités de nature se dégagent à l'analyse : la nature de la présence, la nature du lien social, et enfin la nature de l'engagement. Notre conclusion principale est l'apparition dans le monde du travail d'une nouvelle forme

1 Caroline Sauvajol-Rialland est maîtresse de conférence à l'Université catholique de Louvain et directrice d'un cabinet spécialisé en gestion de l'information en entreprise, So Comment.

Recherches en communication, $\mathrm{n}^{\circ} 37$ (2012). 
de mobilisation collective spécifique dans sa nature. Elle constitue une nouvelle opportunité de communication qui, sans se substituer aux typologies de mobilisation traditionnelles dans le monde du travail, vient se rajouter à elles. Elle apparaît comme fortement liée à l'avènement de la génération née entre 1985 et 1995 que le professeur et chercheur américain Mark Prensky a baptisé, dans un essai désormais célèbre, « Digital Native » (Prensky, On the Horizon, 2001), les véritables « autochtones » du web.

En France, en 2011, deux entreprises du secteur de la grande distribution (Monoprix et Cora) ont, sous la pression de mobilisations «éclair » menées sur les réseaux sociaux, renoncé à des décisions managériales fortes, le licenciement de deux salariés respectivement réassortisseur et caissière. Une autre entreprise du secteur du prêt-à-porter (Kookaï) a subi le même type de «bad buzz » suite au licenciement d'une vendeuse, sans pour autant revenir sur sa décision initiale. Ces événements sont sans précédent dans le monde du travail, en particulier dans le secteur d'activité de la grande distribution qu'on peut difficilement suspecter de « faiblesse » managériale. Si les experts s'accordent aujourd'hui à dire que l'outil numérique a changé les formes de la mobilisation (aspect technique), les récents développements de la recherche sur le sujet de la participation citoyenne sur les réseaux sociaux mettent en garde contre ce que la doctorante canadienne S. Rodriguez nomme la « fétichisation des nouvelles technologies » (Nadeau, 2010, p. 6) et montrent que c'est moins l'outil en soi qui est en train de transformer cette participation que notre façon d'interagir qui est en train de se transformer. A cet égard Internet ne serait pas venu inventer un autre monde virtuel dont le fonctionnement obéirait à d'autres règles que celles du monde réel. Sa contribution propre serait de rajouter à notre expérience sociale une couche technique supplémentaire. Au-delà des différences de formes, existe-t-il une véritable différence de nature entre les formes de mobilisation sur les réseaux sociaux numériques et les formes traditionnelles de mobilisation collective dans le monde du travail ?

$\mathrm{Si}$ ce mouvement de mobilisation dans le monde du travail s'inscrit dans la lignée des mobili-sations collectives à des fins politiques et militantes (Révolutions - ou intifadas - dans le monde arabe, Hacktivistes (contraction de " hacker » - ou pirate informatique - et activiste) de tous ordres, et notamment les Anonymous, et autres Cyber-militants) dans ses effets (relative efficacité), il serait néanmoins différent. Il nous semble ainsi que l'on constate l'apparition d'une nouvelle forme de 
mobilisation collective dans le monde du travail, spécifique dans sa nature et dans ses modalités (impact, origines de la mobilisation, durée de la mobilisation, anonymat, etc..).

\section{Méthodologie}

Afin de démontrer cette hypothèse, nous utiliserons une démarche descriptive et exploratoire des événements visant à raconter le déroulement des trois cas - ou « affaires » - (Monoprix, Cora et Kookaï par ordre chronologique puis par le résultat obtenu par l'action collective entreprise, réussite ou échec) incluant un rappel des faits ainsi qu'un descriptif de la façon dont la médiatisation sous toutes ses formes s'est mise en route. Une analyse des revues de presse dédiées à ces trois affaires a aussi été réalisée et sera présentée parallèlement à la description des faits. Enfin, en dépit du manque de recul dont nous disposons actuellement tant sur ces événements extrêmement récents que sur les réseaux sociaux numériques dont le développement, sinon la naissance, est également récent, une revue de littérature a été effectuée.

\section{Résultats}

Mercredi 6 juillet 2011, Kader en charge de la mise en rayon des produits dans un Monoprix de Marseille est mis à pied et susp,endu à titre conservatoire et, de fait, sous la menace d'un licenciement pour avoir récupéré six melons et deux salades dans la benne à ordures du super-marché. A-t-il été contrôlé « positif» à la sortie du magasin ? A-t-il été repéré puis dénoncé par un cadre aux agents de la sécurité comme le précise un élu CGT ? La marchandise était-elle réellement destinée à être jetée ? Le règlement intérieur précise qu'un salarié « ne doit pas emporter ou utiliser de la marchandise destinée à être jetée » (Direction Générale de la Prévention et des Risques, 2011). En tout état de cause, Kader, 59 ans, $1.1000 €$ par mois et 8 années chez Monoprix est coupable et quasiment condamné. Dévoilée le 7 juillet 2011 dans les colonnes de La Provence sous le gros titre « Menacé de licenciement pour des fruits récupérés dans la poubelle » (La Provence, 7 juillet 2011), la désormais «Affaire Monoprix » est reprise et unanimement commentée par toute la presse nationale : "Un salarié de Monoprix suspendu pour avoir récupéré des aliments »(France Soir, 7 juillet 2011). " Monoprix se comporte en ordure. »(L'Humanité, 8 juillet 
2011) "Kader mis à pied pour 6 melons : un vrai retour au Moyen Age !»(Le Nouvel Observateur, 8 juillet 2011) Et bien sûr Kader se défend : «Quand je suis passé devant la poubelle du magasin, j'ai vu ces fruits qui allaient être jetés. Je les ai trouvés en bon état. J'ai décidé de les rapporter à la maison. C'était juste pour les manger » ( $L a$ Provence, 7 juillet 2011). La direction du Monoprix doit rendre sa décision le 8 juillet 2011. Facebook se met aussitôt en ordre de bataille. Un groupe « Kader ne doit pas être licencié par Monoprix » est créé le 7 juillet 2011 et rassemble rapidement des centaines de participants. La mobilisation est rapide et efficace. « Nous partîmes cinq cents [...] Nous nous vîmes trois mille. " (Corneille, 1637) Les messages de soutien sont nombreux. Untel prône une journée zéro client chez Monoprix. D'autres attribuent des cartons rouges ou incitent les «fans » du groupe à manifester devant les portes du magasin. Au même moment, la page Facebook de la marque est aussi visitée et son mur reçoit tout autant son lot de commentaires parfois ironiques et souvent mécontents. Monoprix attendra une journée mais finira par prendre officiellement la parole par la voix de son Community Manager.

«A tous, nous entendons toutes vos remarques et les regardons avec la plus grande attention [...] Si nous ne pouvons commenter pour le moment l'affaire qui touche notre salarié, nous pouvons vous assurer qu'elle est analysée avec le plus grand soin par le Groupe Monoprix. » (www.facebook.com/Monoprix)

Kader ne sera pas licencié par son entreprise mais uniquement mis à pied une journée pour ne pas avoir respecté le règlement intérieur. Il a repris son travail le vendredi 8 juillet tandis que le " Groupe Kader » l'assurait encore de son soutien indéfectible avant de se féliciter pour une action collective finalement gagnante. De son côté, le Community Manager profitait de la page de Monoprix pour faire part de la situation aux « fans » et surtout surfer sur le «buzz » - une augmentation évidente du nombre de visites durant quelques jours - et récolter quelques fruits. Il a fait preuve de pédagogie en expliquant les principes de sécurité alimentaire visant à éviter la consommation de produits périmés et aussi assuré la publicité de sa marque en rappelant sa collaboration avec des associations comme la Banque Alimentaire ou le Secours Populaire. « Le développement des dons à des associations est un engagement pour Monoprix » indique-t-il (www.facebook.com/Monoprix). 
Les faits sont relativement similaires avec l'hypermarché Cora de Mondelange en Moselle. Anne-Marie, caissière à $940 €$ par mois et élue au comité d'entreprise, ramasse le 28 septembre 2011 un ticket de caisse oublié par une cliente. Une offre de promotion pour un fast-food local s'affiche au dos de ce ticket. "Deux hamburgers achetés : un gratuit ! » Anne-Marie a un fils de 8 ans et décide donc de conserver ce banal bout de papier pré-découpé. Le 26 octobre 2011, Le Républicain Lorrain « sort » l'affaire (Le Républicain Lorrain, 26 octobre 2011). Pour une promotion sur un hamburger, Anne-Marie a été sermonnée par sa chef de caisse puis conduite dans un local par l'adjoint à la sécurité du magasin qui lui a expliqué pendant une heure que ce ticket est la propriété du magasin. Sur les conseils de son avocat, elle s'est ensuite rendue à la gendarmerie d'Uckange pour porter plainte pour séquestration et apprendre qu'elle-même faisait l'objet de poursuites pour vol et serait bientôt convoquée à un entretien préalable de licenciement. L'article est repris le même jour sur le site Internet de L'Express (L'Express, 26 octobre 2011) et sur le réseau social Twitter. La mobilisation est en marche... Anne-Marie est-elle une voleuse ? Faux ! répond sur Twitter Maître Eolas, avocat blogueur de renom, qui précise « qu'un ticket de caisse est la propriété du client dès l'instant où on lui tend. S`il 1)abandonne c>est une res derelicta, chose sans maitre » (https://twitter. com/Maitre_Eolas). Le " buzz » débute le jour même sur Facebook et Twitter. Il va durer 48 heures. Le slogan de Cora « Cora et moi : la confiance est là » devient tweet et déclare haut et fort «Cora et moi : direction le Pôle Emploi ». Les réactions des internautes ressemblent aux réactions des internautes mobilisés contre Monoprix. La page Facebook de la marque devient «The Place to be ». Twitter prend le relais. L'action se joue d'un réseau social l'autre. Une fois encore, la tendance oscille entre humour ("Règlement de compte à OK Cora ») et ironie ( $"$ Et pour un vol de pommes, c'est la chaise électrique ?»). Certaines personnes n'hésitent pas à injurier - en tout anonymat - Cora, le directeur, l'adjoint à la sécurité et la chef de caisse ( « Les pires des $[\ldots] \gg$ ou à réclamer un boycott de l'enseigne voire à proposer à Cora de suivre un cours accéléré sur la responsabilité sociale des entreprises.

«La direction de Cora Mondelange a décidé de ne pas poursuivre la procédure engagée à l'encontre d'une salariée du magasin. Nous avons conscience de l'émotion suscitée par les informations parues depuis ce matin. » (www.facebook.com/hypermarches.cora) Posté le 26 octobre à $19 \mathrm{~h} 38$, ce bref communiqué marque une volte-face de l'entreprise face au tollé engendré par sa décision initiale. Cela n'aura duré 
qu'une journée entre l'annonce publique de l'affaire et la volte-face de Cora. Et Cora de diffuser sur ses pages Facebook et YouTube quatre jours plus tard une vidéo de quelques minutes intitulée « La vie chez Cora, c'est surtout ça... ». Les salariés semblent ravis et disent tout le bien qu'ils pensent de leur entreprise. La réaction du web est unanime ou presque. Rejet ! Et double « bad buzz » pour Cora. Les réseaux bruissent encore pour quelques heures de plus... puis se taisent, définitivement. L'affaire Cora est terminée pour ce qui concerne son volet web. Le volet judicaire se poursuit encore car Anne-Marie, soutenue par la CGT qui depuis le début considère que l'affaire Cora n'offre que prétexte à chasser les représentants syndicaux, n'a jamais accepté d'être assimilée à une « voleuse » et poursuit son employeur pour " dénonciation calomnieuse », une autre histoire qui ne semble pas affoler la toile... pour le moment.

Le 9 avril 2011, Nadine Morano, ministre chargée de l'Apprentissage et de la Formation professionnelle, fait du shopping au Printemps de Nancy en compagnie de sa fille et de son garde du corps. Albane a 36 ans et la responsabilité du stand Kookaï, marque de prêt-à-porter féminin. Evoquant la présence du bodyguard dans le magasin, une vendeuse du magasin explique que l'un de ses collègues a affirmé sur le ton de la plaisanterie « qu'il pourrait le mettre en deux temps, trois mouvements par terre ». Et Albane d'ajouter : « que pour se mesurer à lui, il fallait d'abord casser la gueule à madame Morano » (rue89.com, 30 mai 2011). Nadine Morano se trouvait à proximité d'Albane qui s'excuse immédiatement. En vain, la ministre réclame la venue d'un responsable et les coordonnées du directeur du Printemps et promet " des suites à ce dossier" (rue89.com, 30 mai 2011). De son côté Albane décide de contacter la responsable régionale de Kookaï pour lui expliquer ce qu'il s'est passé. Le lundi 11 avril, la vendeuse est mise à pied à titre conservatoire, reçoit finalement une convocation pour un entretien à Paris le 21 avril puis est licenciée pour faute grave. «Votre attitude nous a résolument choqués ; vous avez non seulement jeté l'opprobre sur la société Kookaï auprès de l'une de nos clientes mais aussi terni l'image de la société dans ses relations de partenariat avec le Printemps » mentionne la lettre commandée (rue89.com, 30 mai 2011) adressée à Albane le 26 avril 2011. Interrogée par France Info le 31 mai 2011, Nadine Morano n'estime pas être à l'origine de ce licenciement mais donne cependant sa propre version des faits en rapportant que la vendeuse aurait dit à qui voulait l'entendre que « Nadine Morano [est] dans le magasin, si quelqu'un veut aller lui casser la gueule... » (France 
Info, 31 mai 2011). A l'image de ce qui s'est produit pour Monoprix et de ce qui se produira pour Cora, une action de protestation collective s'est mise en place sur Facebook le jour de la « sortie » de l'affaire sur rue89.com, principalement sur la page Facebook de la marque. Un

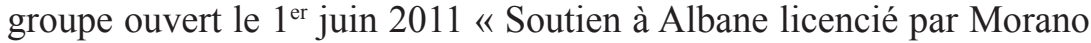
Boycott Kookaï ! » n'a pour sa part récolté qu'une audience intime et peu de commentaires. L'information finalement était sur la page officielle. Chaque discussion pro-posée sur le mur était " enrichie » de nombreux commentaires sur l'affaire, des commentaires vindicatifs. Le Community Manager de la marque s'est alors empressé de censurer ses « fans » en supprimant leur message et de leur rappeler la seule et unique vocation de cet espace de communication, évidemment corporate, dans un post sans équivoque.

Cette page Facebook est destinée à informer les fans de la marque des bons plans, des nouveautés de la collection, des jeux concours et des coups de cœur à partager. [...] Nous remercions par avance chacun de respecter cet espace et sa raison d'être première. Pour toute autre demande ou commentaire, merci d'adresser un courrier au siège Kookaï. (www.facebook.com/ kookai)

Effet boomerang et re-révolte de toute la communauté et puis... Silence. En avril 2012, Albane a trouvé un accord financier avec son ancien employeur, pas un nouvel emploi. Le web pour le moment n'a pas réagi et ne le fera sans doute plus.

Il est intéressant de noter que cette mobilisation s'est faite au moyen des réseaux sociaux pas spécifiquement professionnels (sur Facebook et Twitter et non sur Viadeo ou LinkedIn).

\section{Discussion}

Les différences de forme entre mobilisation collective traditionnelle et mobilisation collective virtuelle sont connues. Par leur gratuité - au moins en version « light » pour certains -, par la facilité à les comprendre et à s'en servir et par leur rapidité, les réseaux sociaux numériques permettent la structuration et l'organisation. Internet comme « thermomètre social » est devenu un véritable outil d'action. Les réseaux sociaux permettent d'agir en mode collectif avec les autres avec une facilité qui 
aurait été inconcevable il y a 15 ans à peine... Au-delà de ces différences de forme, quelles sont les différences de nature?

\section{La nature « présence » physique ou virtuelle}

Dans le monde physique, il existe une unité inhérente au moi, le corps donnant en effet une définition efficace et adéquate de l'identité. La norme institue : un corps, une identité. Bien que le moi puisse être complexe et changeant à travers le temps et les circonstances, le corps assure un ancrage stable. Sartre écrivait dans L'être et le néant : « Je suis mon corps dans la mesure où Je suis. » Le monde virtuel est différent, composé d'information plutôt que de matière. Les peuplades de cet espace évanescent sont tout autant diffuses, libres de l'ancrage du corps. D'aucun pense que chacun peut avoir au-tant d'avatars électroniques que de temps et d'énergie à consacrer pour leur création. (Donath, 1999)

Le réseau social serait alors « une catégorie de site web avec des profils d'utilisateurs, des commentaires publics semi persistants sur chaque profil, et un réseau social public navigable affiché en lien direct avec chaque profil individuel » donc une « collection de profils» (boyd, 2007), soit une liste de pseudonymes reflétant la réalité physique de la personne. La mobilisation sur les réseaux sociaux est « virtuelle ». L'identification des émetteurs est floue. Qui est par exemple à l'origine de tel ou tel «buzz »? Chacun s'engage de façon anonyme. Et pour le moment cette forme de mobilisation n'a pas débouché sur des actions dans le monde réel (manifestations) à l'image de ce qui s'est passé dans le monde arabe au moment des intifadas. Si l'on revient sur le cas particulier de Kookaï, le licenciement de la salariée est finalement devenu effectif sans que ne se remobilisent une fois encore les réseaux sociaux.

\section{La nature du lien social}

Selon S. Wasserman et K. Faust, le réseau social permet de mettre en relation des milliers d'individus autour des mêmes centres d'intérêt ou non, de les faire échanger des informations ou encore de les faire participer à des évènements, le tout à distance et sans contacts réels (Wasserman \& Kraut, 1994). Il existe une filiation naturelle entre les réseaux sociaux numériques et les réseaux sociaux traditionnels. Les analogies possibles concernent aussi bien les techniques d'analyse, les 
mesures et les notions applicables que la conceptualisation en termes de structures sociales, interdépendances, capital relationnel et statut social des individus. Un réseau social numérique attaché à une personne ne représente-t-il finalement qu'une copie du réseau traditionnel « off line » de cette même personne ou bien est-il une composante séparée ? Et finalement le réseau en ligne peut-il rétroagir sur son réseau hors ligne en en modifiant la taille, la composition, ou même la structure ? Pour Robert Kraut et ses co-auteurs il existe une relation négative entre la construction et le maintien des liens sur Internet, généralement avec des personnes éloignées physiquement et affectivement (liens faibles), et l'entretien de relations de proximité avec son entourage familial et amical (liens forts) : une technologie supposée socialisante se révélerait alors affaiblir le lien social. Cette vision a ensuite été nuancée par les auteurs eux-mêmes après réexamen des données sur une plus longue période (Kraut et al., 2012). La perspective dominante actuellement est plutôt celle de B. Wellman et L. Rainie qui voient Internet comme un outil additionnel pour aider la sociabilité et considère les liens en ligne et hors ligne comme complémentaires plutôt que substituables (Wellman \& Rainie, 2012). Fabien Granjon a pour sa part interrogé la notion d' « ami » sur Facebook (Granjon, 2011). Il fait apparaître la variété et la complexité des relations humaines qui se cachent sous le terme générique d'ami. L'engagement se crée par la sollicitation d'amis et non par une volonté délibérée et préalable. Il n'existe pas de véritable communauté constituée et plus un système de combustion et de contagion qu'un véritable militantisme. Les réseaux sociaux numériques font office de catalyseurs, d'amplificateurs et d'accélérateurs. Ils sont un outil d'influence. Ils sont l'incarnation d'une coalition provisoire et spontanée échappant par là même à la communauté. Facebook favorise l'émergence, la tenue, de mobilisations éphémères, de «flash mob » de la contestation qui se désagrègent aussi vite qu'ils se sont cristallisés.

\section{La nature de l'engagement}

On pensait que les jeunes étaient beaucoup moins engagés que leurs aînés et qu'ils se désinté-ressaient de l'action collective.

On note une baisse de la participation dans les mouvements sociaux dits traditionnels tels que les syndicats ou les partis politiques. Mais ce ne sont pas nécessairement les jeunes qu'il faut blâmer. « Il y a une transformation de l'action collective 
à l'échelle internationale et les jeunes ne sont que le reflet de cette mutation » estime [S. Rodriguez]. [...] Devant la rapidité des nouvelles technologies, les jeunes voient de plus en plus les mouvements sociaux traditionnels comme des moyens usés, inefficaces et lourds. « Les jeunes trouvent important de voter et de s'engager, reprend S. Rodriguez, mais dès qu'on leur parle de syndicat, de partis politiques ou de structures organisées ou hiérarchiques, ils ont tendance à dire que ce n'est pas très efficace. » Ils [les jeunes] font des choix personnels et de responsabilités individuelles, de choix éthiques de travail et de consommation. Leurs engagements se font plus distancés, multiples et ponctuels. (Nadeau, 2010, p. 6)

Désormais des masses qui semblent en apparence sans chef et ne sont pas organisées sous la forme traditionnelle du parti s'emparent d'outils de communication et en font des armes de contestation efficaces. Internet a rendu possible la conciliation entre engagement collectif et liberté individuelle, tout en conservant l'efficacité. S'agit-il d'une forme d'individualisme exacerbé ou une nouvelle forme d'engagement sélectif? En tout état de cause, « les technologies favorisent en particulier dans la sphère de la relation à l'autre et à l'autorité publique, religieuse, familiale, des attitudes, des représentations, des pratiques toujours plus autonomes et individualisées qui rendent à l'évidence aujourd'hui impossible ou presque le maintien des anciennes formes de légitimité » Gonzalez-Quijano, 2011). Selon Miguel Benasayag, militer était un combat, un sacerdoce, c'est devenu un plaisir (Benasayag, 2010, pp. 9-12). Michel Chrestien, personnage des Illusions perdues, républicain intègre et passionné, en était 1'archétype (Balzac, 1837). Il s'agit donc plutôt d'une mobilisation autour d'une indignation que d'un militantisme profond ou la défense de valeurs partagées. C'est une addition/ multiplication de contestations d'individus qui n'ont pas forcément en commun de grande cause mais une volonté de lutte contre l'injustice, de faire changer les choses. Leur attitude est réactive et épidermique. Les personnes concernées par les procédures de licen-ciements occupaient des postes pénibles et peu rémunérateurs. Une certaine compassion en découle et provoque une contagion des émotions. Les réseaux sociaux agissent en meute. De simples commentaires se transforment en quelques minutes en un véritable rouleau compresseur. Un frémissement apparaît, parfois imperceptible, puis se trans-forme en phénomène de combustion spontanée. Pour P. Lévy, « Les réseaux sociaux ont une vertu libératrice » (Lévy, 2002). Mais « l'individu reste fidèle à lui- 
même. Une fois que l'action ou l'événement n'est plus d'actualité, le pseudonyme va migrer vers d'autres groupes, mais ces derniers gardent un lien perceptible avec le mobile d'origine » précise $M$. Guidère (Castagnac, 2011).

Toutefois, en retour, « ces outils favorisent des manières de penser en commun, de décider, de lutter (elles suscitent des stratégies et des contre stratégies de l'adversaire) donc ils transfor-ment ce qu'il faut entendre par engagement ou révolte » (Huygue, 2011).

\section{Conclusion}

Nous avons pu constater l'apparition dans le monde du travail d'une nouvelle forme de mobilisation collective spécifique dans sa nature. Elle est le fait d'un changement générationnel, autant que de la mise à disposition de nouveaux outils. Si, au plan technique, il ne s'agit que d'une application au monde du travail d'une technologie jusque-là applicable dans les univers privés puis politiques, elle constitue une nouvelle opportunité de communication qui, sans se substituer aux typologies de mobilisation traditionnelles dans le monde du travail, vient se rajouter à elles. Les réseaux sociaux numériques découvrent peu à peu leur pouvoir d'influence - et nous découvrons ce pouvoir en même temps que nous l'observons. Ils sont en capacité de faire changer d'avis une direction d'entreprise, de lui « imposer » leur décision et « invalider » la leur. Un nouveau rapport de force est en train de s'instaurer. Par ailleurs, les réseaux sociaux apparaissent aussi comme un nouveau vecteur d'information pour les médias traditionnels. Ils les interpellent... Tout comme les réseaux sociaux interpellent par la suite les hommes et les femmes politiques. Au sujet de l'affaire Cora, comme l'ont rapporté de nombreux sites de journaux et notamment Libération dans son édition du 27 octobre 2011, Aurélie Filipetti, alors députée de Moselle, dénonçait « une mascarade honteuse » tandis qu'Europe Ecologie Les Verts invitait Cora à « mettre un terme à cette procédure absurde » (Libération, 27 octobre 2011). " Il est évident qu'Internet change nos façons de vivre ensemble et a fortiori sous sa forme que l'on a baptisé 2.0 (Le terme Web 2.0 a été mis au point en 2004 par D. Dougherty (O’Reilly Media) et C. Cline (MediaLive) pour caractériser la conférence qu'ils s'apprêtaient à initier - Web 2.0 Conference) qui repose sur le principe de " tous émetteurs, tous connectés, tous coopérant » et sur le « partage » que ce soit des vidéos, des expé- 
riences quotidiennes ou des indignations révolutionnaires »( Huygue, 2011). Nous observons que cette constatation est valable aussi dans le monde du travail, par extension. Nous sommes dans une forme d'action citoyenne appliquée au monde du travail. Désormais, l'ensemble du corps social s'est emparé du web 2.0 pour faire porter sa voix plus loin, plus haut. Reste que les conséquences d'un « bad buzz » ou boucheà-oreille négatif peuvent être importantes et durables. Le monde de la grande distribution - largement et souvent - vilipendé pour ses pratiques commerciales et de management a " payé pour voir » avec l'affaire Cora. Cela vaut avertissement pour l'ensemble des autres grandes enseignes du marché... L'entreprise se doit de surveiller la toile. L'enjeu est simple. Il peut en aller de sa réputation et de son image. Pourtant ces organisations ont encore du mal tant à entrer dans une véritable conversation avec les réseaux sociaux numéri-ques qu'à faire montre d'une certaine transparence. Il lui faut pourtant se préparer à répondre de ces décisions et de ses actes auprès de tous. Quoi qu'il en soit ce champ de recherche est extrêmement jeune. Il est en pleine effervescence, "remué » par une accumulation exponen-tielle d'expériences et de connaissances nouvelles, et nous ne disposons que d'un faible recul temporel sur les événements. La mobilisation virtuelle évoluerat-elle vers des mobilisations dans le monde réel comme ce fut le cas lors du « Printemps arabe », évoluera-t-elle vers le domaine politique? Ou bien en restera-t-elle à l'espace virtuel ? Par ailleurs, il serait utile d'interroger les nouvelles relations entre les médias traditionnels et les nouveaux médias numériques. Les médias traditionnels sont pris de court par les médias numériques qui devien-nent pour eux une immense base de données, d'informations. Réunis, leur puissance semble extraordinaire. Pour M. Benasayag « les modèles ont disparu mais les causes demeurent. Le sens de l'action n'est plus donné d'avance. Il se construit au quotidien en tâtonnant. Il s'agit de lutter sans modèle » (Benasayag, Les idées en mouvement, 2010, pp. 9-12).

\section{Références}

Balzac, H. (1837). Illusions perdues. Paris : Le Livre de Poche 2006, coll. Classiques. Benasayag, M. (2010). Nouveaux militants : réinvention du politique ? Les idées en mouvement, 184, pp. 9-12.

Boyd, D. (2008). Why Youth (Heart) Social Network Sites: The Role of Networked Publics in Teenage Social Life. Dans David Buckingham (Ed.), Youth, Identity, and Digital Media, The John D. and Catherine T. MacArthur Foundation Series on Digital Media and Learning, pp. 119-142. Cambridge, MA: The MIT Press. 
Castagnac, P.-Y.(2011). Tunisie, Egypte, Libye : A chaque révolution sa communication, entretien avec Mathieu Guidère, professeur titulaire de la chaire d'Islamologie et pensée arabe à l'Université de Toulouse II. In Huygue F.-B. (Ed.), Facebook, Twitter, Al-Jazeera et le "Printemps arabe ». Paris : Institut des Relations Internationales et Stratégiques.

Corneille, P. (1637, 2004). Le Cid. Paris: Editions Gallimard, Coll. Folio Classiques.

Direction Générale de la Prévention et des Risques (2011). Rapport intermédiaire de l'étude relative au gaspillage alimentaire. Ministère de l'Ecologie, du Développement durable, des Transports et du Logement.

Donath, J. (1999) Identity and deception in the virtual community. In Kollock P. \& Smith M. (Eds.), Communities in Cyberspace: Perspectives on New Forms of Social Organization, pp. 27-57. London : Routledge.

El Oifi, M. (2011). L'autonomisation des opinions publiques. In Huygue F.-B. (Ed.), Facebook, Twitter, Al-Jazeera et le "Printemps arabe ». Paris : Institut des Relations Internationales et Stratégiques.

Gonzalez-Quijano, Y. (2011). « Les origines culturelles numériques » des révolutions arabes. Paris : Sciences-Po, Ceri-CNRS.

Granjon, F. (2011). Amitiés 2.0. Le lien social sur les sites des réseaux sociaux. Hermès, 59, pp. 99-104.

Huygue, F.-B. (Ed.) (2011). Facebook, Twitter, Al-Jazeera et le "Printemps arabe ». Paris : Institut des Relations Internationales et Stratégiques.

Kraut, R. et al. (2012). Building Successful Online Communities: Evidence-Based Social Design. Cambridge, MA : The MIT Press.

Lévy, P. (1997). L'intelligence collective, pour une anthropologie du cyberspace. Paris : La Découverte.

Lévy, P. (2002). Cyberdémocratie. Paris : Editions Jacob.

Nadeau, J. (2010). S'engager à l'ère du Web 2.0. Perspectives CSN, Dossier Travail et militance : tendances et défis, 30,6.

Prensky, M. (2001). Digital Natives, Digital Immigrants Part 1. On the Horizon, 9(5), $1-6$.

Stenger, T., \& Coutant, A. (Eds.) (2011). Ces réseaux numériques dits sociaux [Numéro thématique]. Hermès, 59.

Wasserman, S., \& Faust, K. (1994). Social Network Analysis: Methods and Applications. Cambridge: Cambridge University Press.

Wellman, B., \& Rainie, L. (2012). Networked. The New Social Operating System. Cambridge, MA: The MIT Press.

\section{Sources : articles de presse}

\section{Affaire Monoprix}

Comenge Y. (2011). Kader mis à pied pour 6 melons : un vrai retour au Moyen Age ! In Le Nouvel Observateur, 8 juillet 2011. Disponible à :leplus.nouvelobs.com/ contribution/168962-kader-mis-a-pied-pour-6-melons-glanes-un-vrai-retour-aumoyen-age.html

D’Ancona L. 2011. Menacé de licenciement pour des fruits récupérés dans la poubelle. In La Provence, 7 juillet 2011. Disponible à : www.laprovence.com/article/ 
marseille-176

M. L. (2011). Monoprix se comporte en ordure. In L'Humanité, 8 juillet 2011. Disponible à : http://www.humanite.fr/07_07_2011-monoprix-se-comporte-en-ordure-475998

Un salarié de Monoprix suspendu pour avoir récupéré des aliments. In France Soir, 7 juillet 2011. Disponible à :http://www.francesoir.fr/actualite/societe/un-salariemonoprix-suspendu-pour-avoir-recupere-des-aliments-116474.html

\section{Affaire Cora}

Menacée de licenciement pour un ticket de caisse ramassé. In L'Express, 26 octobre 2011. Disponible à : www.lexpress.fr/emploi-carriere/emploi/menacee-delicenciement-pour-un-ticket-de-caisse-ramasse_1044711.html

Mondelange : menacée de licenciement pour un ticket de caisse. In Le Républicain Lorrain, 26 octobre 2011. Disponible à : www.republicain-lorrain.fr/ actualite/2011/10/26/menacee-de-licenciement-pour-un-ticket-de-caisse

Une caissière risque le licenciement pour avoir ramassé un ticket de caisse. In Libération, 26 octobre 2011. Disponible à : www.liberation.fr/societe/01012367909-caissierecora-licenciement-coupon-reduction

Caissière accusée de vol : Cora abandonne la procédure de licenciement. In Libération, 27 octobre 2011. Disponible à : www.liberation.fr/societe/01012368093-caissiereaccusee-de-vol-cora-abandonne-la-procedure-de-licenciement

Cora : La caissière « sauvée » grâce au soutien sur Internet. In France Soir, 27 octobre 2001. Disponible à : http://www.francesoir.fr/actualite/societe/cora-caissiere-sauveson-emploi-grace-au-soutien-sur-internet-151122.html

\section{Affaire Kookaï}

Cerveaux A. (2011). Elle plaisante sur Morano et est licenciée pour faute grave. In rue89.com, 30 mai 2011. Disponible à : http://www.rue89.com/2011/05/30/elleplaisante-sur-morano-et-est-licenciee-pour-faute-grave-206765

\section{Réseaux sociaux numériques}

www.facebook.com/Monoprix https://twitter.com/Maitre_Eolas www.facebook.com/hypermarches.cora www.facebook.com/kookai 